\title{
Validation of organ dose calculations with PyMCGPU-IR in realistic interventional set-ups
}

\author{
David Fernández-Bosman $^{\mathrm{a}, *}$, Ariel von Barnekow ${ }^{\mathrm{a}}$, Jérémie Dabin ${ }^{\mathrm{b}}$, Françoise Malchair ${ }^{\mathrm{c}}$, \\ Filip Vanhavere ${ }^{\mathrm{b}}$, Maria Amor Duch ${ }^{\mathrm{a}}$, Mercè Ginjaume ${ }^{\mathrm{a}}$ \\ ${ }^{a}$ Universitat Politècnica de Catalunya, Avda. Diagonal 647, 08028 Barcelona, Spain \\ ${ }^{\mathrm{b}}$ Belgian Nuclear Research Centre (SCK CEN), Boeretang 200, $2400 \mathrm{Mol}$, Belgium \\ ${ }^{\mathrm{c}}$ ZEPHYRA, Rue Forgeur 13, 4000 Liege, Belgium
}

\section{A R T I C L E I N F O}

\section{Keywords:}

Monte Carlo

Interventional procedures

Dose distribution

Execution time

Voxel phantom

\begin{abstract}
A B S T R A C T
Introduction: Interventional radiology procedures are associated with high skin dose exposure. The 2013/59/ EURATOM Directive establishes that the equipment used for interventional radiology must have a device or a feature informing the practitioner of relevant parameters for assessing patient dose at the end of the procedure. This work presents and validates PyMCGPU-IR, a patient dose monitoring tool for interventional cardiology and radiology procedures based on MC-GPU. MC-GPU is a freely available Monte Carlo (MC) code of photon transport in a voxelized geometry which uses the computational power of commodity Graphics Processing Unit cards (GPU) to accelerate calculations.

Methodologies: PyMCGPU-IR was validated against two different experimental set-ups. The first one consisted of skin dose measurements for different beam angulations on an adult Rando Alderson anthropomorphic phantom. The second consisted of organ dose measurements in three clinical procedures using the Rando Alderson phantom.

Results: The results obtained for the skin dose measurements show differences below 6\%. For the clinical procedures the differences are within $20 \%$ for most cases.

Conclusions: PyMCGPU-IR offers both, high performance and accuracy for dose assessment when compared with skin and organ dose measurements. It also allows the calculation of dose values at specific positions and organs, the dose distribution and the location of the maximum doses per organ. In addition, PyMCGPU-IR overcomes the time limitations of CPU-based MC codes.
\end{abstract}

\section{Introduction}

In Interventional Radiology (IR) procedures, real-time images are obtained by generating a large number of consecutive single X-Ray images. Despite the fact that the radiation dose used for a single image being low, the cumulative dose can sometimes lead to high skin dose exposure [1]. The 2013/59/EURATOM Directive [2] establishes that the equipment used for interventional radiology must have a device or a feature informing the practitioner of relevant parameters for assessing patient dose at the end of the procedure. ICRP 105 [3] also pointed out the fact that there is a need for real-time monitoring whether the threshold doses for deterministic effects are either being approached or exceeded for a particular patient, as the absorbed dose in the skin in the area of maximum cumulative skin dose is the quantity of major concern for fluoroscopically guided interventional procedures. According to the ICRP report, a statement on cumulative skin doses and skin sites should be included in the clinical protocol.

Several software tools have been developed for the skin dose calculation (SDC) in order to provide the peak skin dose (PSD) during or after the procedure [4]. The approach used by most of SDC tools to estimate the PSD is the same and consists of the methodology proposed by Jones and Pasciak [5]. This method consists of taking dose metrics from the Radiation Dose Structured Report (RDSR), such as the air kerma at the reference point $\left(\mathrm{K}_{\mathrm{a}, \mathrm{r}}\right)$ or the kerma-area product (KAP), and then converting them to PSD by applying a set of correction factors to account for different physical phenomena, such as back scatter or table attenuation.

A completely different approach is to use the Monte Carlo (MC) method to simulate radiation transport. Monte Carlo simulations

\footnotetext{
* Corresponding author.

E-mail address: david.fernandez.bosman@upc.edu (D. Fernández-Bosman).
} 
provide a wide range of advantages. The simulations are useful not only in the calculation of the PSD, where the simulations already account for all the physical phenomena and thus there is no need to use correction factor, but also because the dose can be calculated both in different tissues and organs. However, the MC method is generally very time consuming and this has been the main reason that it has been left aside as an SDC tool. This problem can be solved by using the computational power of the graphics processing units (GPUs). Using GPUs, simulations can be massively parallelized resulting in a drastic decrease in computational time.

The Universitat Politècnica de Catalunya (UPC) and the Belgian Nuclear Research Centre (SCK CEN) are participating in Task 2.2.2 of Work-Package 2 of MEDIRAD, a H2020 European Project aimed to enhance the scientific bases and clinical practice of radiation protection within the medical field [6]. The objective of MEDIRAD Task 2.2.2 is to develop a real time patient dose monitoring application for interventional cardiology procedures based on the freely available accelerated MC code, MC-GPU [7]. In particular, the study aims to both validate and improve MC-GPU in order to determine patient skin dose distribution and organ doses for specific realistic clinical set-ups in IR.

In a recently published paper MC-GPU was validated against the well-known PENELOPE/PenEasy code system and against experimental conditions for typical radiation qualities and set-ups in IR [8]. The results showed that MC-GPU is capable of simulating the underlying physical phenomena involved in IR procedures and also of calculating the doses in different tissues and organs in very short computation times (less than one minute per irradiation event). From the perspective of the MEDIRAD Task 2.2.2 objective, and as a continuation of the already mentioned work [8], the next steps for the implementation of MC-GPU in clinics consist in the following. On the first hand, the improvement of MC-GPU to fulfil all the basic requirements needed by an SDC tool, such as automatization of all the steps needed for the dose calculation. On the other hand, to validate MC-GPU against realistic clinical set-ups in IR.

The above-mentioned improvements of MC-GPU for its implementation in clinics have been achieved through the development of the PyMCGPU-IR program [9]. PyMCGPU-IR is a Python [10] application that provides automatic acquisition of the X-ray source conditions from the patient's Radiation Dose Structured Report (RDSR) [11]. The main purpose of this work is to validate PyMCGPU-IR for its use as a patient dose monitoring tool in IR procedures.

\section{Materials and methods}

\section{PYMCGPU-IR}

MC-GPU is a MC simulation code that can generate synthetic radiographic images of realistic models of the human anatomy by using the computational power of commodity GPU cards. The code implements a massively multithreaded MC simulation algorithm for photon transport in a voxelized geometry. The X-ray interaction models and material properties have been adapted from PENELOPE [12]. The code was first developed by Badal et al 2009 [7], but, as has been already highlighted, it was upgraded and validated as described in a previous article [8].

MCGPU-IR was also developed for the calculation of operator's dose in fluoroscopically guided procedures [13]. It is an extension of the original MC-GPU, which also added the possibility to automatically simulate several irradiation events, thus providing the total organ doses together with the individual results for each event. For each irradiation event, all beam relevant parameters such as $\mathrm{kV}$, added filtration, position and angulation of the beam are taken into account. In addition, MCGPU-IR, implemented the calculation of a normalization factor to convert dose values per simulated history to absolute values, for each event. This factor was derived from the experimental measurement of $\mathrm{K}_{\mathrm{a}, \mathrm{r}}$. However, MCGPU-IR was developed to be used together with a specific web application to calculate the radiation worker's dose, not the patient doses. Thus, some technical modules, such as management of the procedure's data, were integrated in the web application and therefore, were not available as a stand-alone code.

As a further step forward, we developed PyMCGPU-IR, a Python application for MCGPU-IR that includes patient dose calculations in interventional radiology. It can read several types of input data files (e.g. RDSR) to retrieve the required information about each irradiation event and transform them into MCGPU-IR input files. The application then proceeds to launch the simulation, and finally retrieves the radiation doses from its output. A schematic diagram of the PyMCGPU-IR workflow is shown in Fig. 1. At present, PyMCGPU-IR is not publicly available. However, a Demo of PyMCGPU-IR has been prepared to show how the developed patient dose calculation tool works [9].

PyMCGPU-IR supports reading the RDSR both in the standard DICOM format and in the spreadsheet format, namely, Microsoft Excel file format (.xls) and Office Open XML file formats (.xlsx) based on Siemens Care Analytics [14]. It also supports its own format in both Microsoft Excel file format (.xls) and Office Open XML file formats (. $\mathrm{xlsx}$ ). Before launching the dose calculation, PyMCGPU-IR extracts the relevant information from the RDSR such as source position, kilovoltage peak, angulation and other information, as shown in Fig. 2.

Despite the fact that the RDSR is a standard, there are some fields, especially those involving the relative position of the patient with respect to the beam source, that are encoded in a way specific to the manufacturer. In addition, in some cases the RDSR does not provide all the required information, either because it is anonymous with, for example, missing patient gender, or it is incomplete because the clinician did not introduce some information, such as patient height or
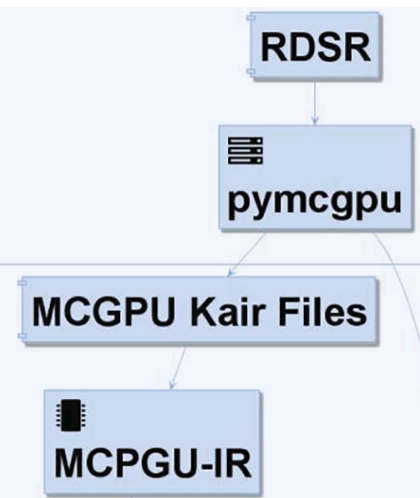

\section{OUTPUT 1 MCGPU Patient Files}

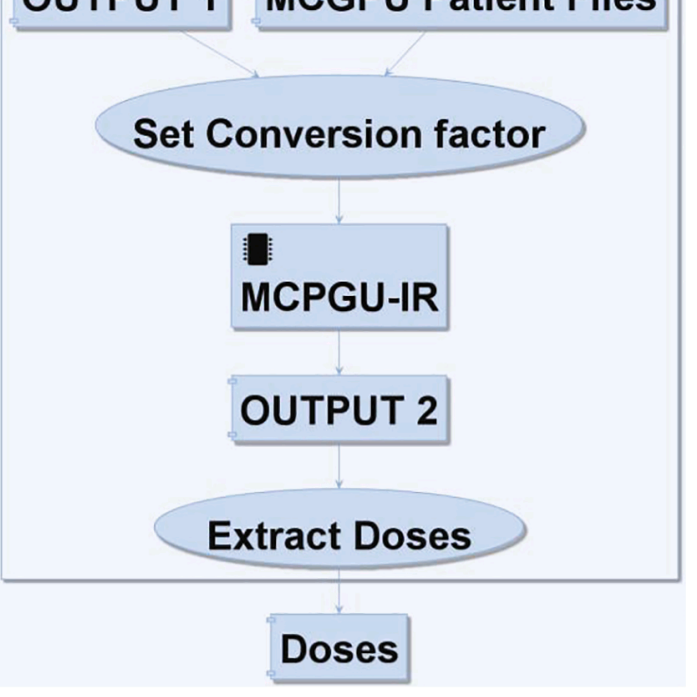

Fig. 1. PyMCGPU-IR work flow. 


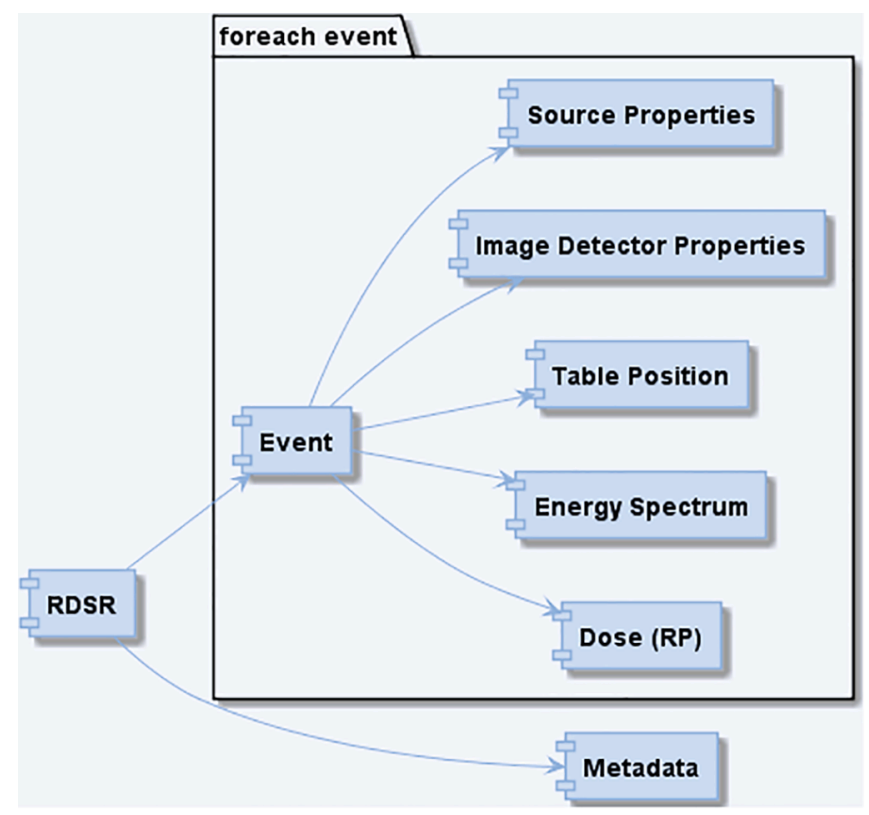

Fig. 2. Relevant data extracted from the RDSR for dose calculation.

weight.

In order to bypass these issues, PyMCGPU-IR supports reading another file which is created by the user and is used to complement the RDSR. This file may contain information about the patient, X-Ray source parameters and the relative position between the patient and the X-Ray source.

In a typical IR procedure, once the X-ray beam is positioned on the region of interest and has been tuned to obtain adequate image quality, it may remain in the same configuration for a set of consecutive single irradiation events with only slight modifications until irradiations from a different direction or a new region of interest are needed This means that there may be sets of events with only slight differences in radiological parameter values. To reduce the calculation time, a clustering algorithm was developed to group these sets of events into single ones, as the slight differences among them lead to negligible differences in terms of organ dose, thus reducing the number of total events to be simulated by MC-GPU. The proposed algorithm groups those events that share similar characteristics under specific criteria into single ones. A trigger value is defined for each parameter. This value indicates the maximum difference that two events can have in order to be grouped together as a single event. Given two events, the difference between the parameter values is calculated. If all the differences are below or equal to the corresponding trigger value, then the events are grouped together. The events are grouped in a consecutive way. When two events are grouped together a group-event (the event that represents the group) is generated and its parameter values are defined as the mean parameter values of both events. The next event will be compared to the different group-events and if it is to be added to a group, the group-event will be updated in the same manner. Table 1 shows the trigger value defined for each parameter, based on a follow-up analysis of typical ranges in interventional radiology.

PyMCGPU-IR models the patient using the voxel adult reference computational phantoms, Rex and Regina, developed by Helmholtz Zentrum München $[15,16]$ for male and female patients, respectively.
PyMCGPU-IR can be configured to work with other voxel phantoms. Rex and Regina have the same anatomy but have a different elemental tissue composition from the ICRP Publication 110 reference phantoms [17]. In PyMCGPU-IR, the 145 segmented objects in the original phantoms are simplified and grouped together into 27 different materials. The Rex voxel dimensions are $0.21 \times 0.21 \times 0.80 \mathrm{~cm}^{3}$ resulting in a voxel volume of $36.53 \mathrm{~mm}^{3}$, while the Regina voxel dimensions are $0.18 \times 0.18 \times$ $0.48 \mathrm{~cm}^{3}$ resulting in a voxel volume of $15.25 \mathrm{~mm}^{3}$. For each material, both the maximum and mean dose can be calculated as well as the location of the maximum dose. In our calculations, the original size of the Rex phantom was used. However, a scaling factor for each dimension of the phantom can be applied. With this scaling factor, the model can be adapted to a particular body size.

\section{Experimental measurements}

Measurements were performed at the Centre Hospitalier Universitaire de Liège in Belgium [18] in a cardiology theatre equipped with a Siemens Axiom Artis angiographic system. Experimental dose assessment was done by using thermoluminescent detectors (TLDs) from the SCK CEN dosimetry service [19]. The TLDs were $0.9 \mathrm{~mm}$ thick an 4.5 $\mathrm{mm}$ diameter cylindrical pellets made of LiF:Mg, Cu, P material (MCP-N, Institute of Nuclear Physics, Poland). Prior to irradiation, the TLDs were annealed at $240{ }^{\circ} \mathrm{C}$ during $10 \mathrm{~min}$ then rapidly cooled in a freezer at $-10{ }^{\circ} \mathrm{C}$. TLDs were used for background monitoring. Before read-out, the dosimeters were heated at $120^{\circ} \mathrm{C}$ for $30 \mathrm{~min}$. The TLDs were read out on a Harshaw 5500 system with a constant rate of $10^{\circ} \mathrm{C} / \mathrm{s}$ from room temperature up to $240{ }^{\circ} \mathrm{C}$. Readings were corrected for the sensitivity of the detectors, which was individually determined using a Cs-137 source. TLDs were calibrated at a primary standard laboratory using a heavily filtered beam representative of IR condition $(80 \mathrm{kVp}, 0.9 \mathrm{~mm} \mathrm{Cu}$ added filtration and $8.63 \mathrm{~mm} \mathrm{Al} \mathrm{HVL}$ ) as described in [20].

Two sets of experiments were designed. A first set of three simple measurements consisted of several TLD arrays placed on the back of the Rando Alderson (RA) anthropomorphic phantom in order to measure both the PSD and skin dose distribution (Section 2.2.1). The second set of measurements consisted of three clinical procedures simulated on the RA phantom with TLDs placed inside it at the level of the lung and esophagus to measure the absorbed organ doses (Section 2.2.2).

\section{Skin dose set-up}

This experiment was intended to measure the skin dose for different beam angulations on a realistic patient representation. To this end, an adult RA anthropomorphic phantom (The Phantom Laboratory, USA), which represents a patient weighing about $75 \mathrm{~kg}$, was irradiated in three simple irradiation set-ups. The first is a Posterior Anterior (PA) irradiation, the second is a combination of PA and Lateral Oblique (PALAO) irradiations and the third is a combination of PA, LAO and Cranial (PALAOCRA) irradiations. The beam energy and both the Aluminum and Copper filtrations for the three irradiation set-ups were $77 \mathrm{kV}, 3 \mathrm{~mm}$ of Aluminum and $0.1 \mathrm{~mm}$ of Copper, respectively. The Crano-Caudal and Lateral beam angulations for the PA, LAO and Cranial irradiations were $\left(0^{\circ}, 0^{\circ}\right),\left(20^{\circ}, 0^{\circ}\right)$ and $\left(0^{\circ}, 15^{\circ}\right)$, respectively.

The RA phantom was positioned on a mattress and the TLD detectors were sited on the mattress beneath the phantom centered on the patient's heart. For the PA irradiation set-up, three TLDs were held in a small bag and for the PALAO and PALAOCRA irradiation set-ups a grid of 25 TLDs was used to ensure that the PSD was recorded. The grid was composed of $5 \times 5$ TLDs with a separation of $3 \mathrm{~cm}$ between each of them

Table 1

Trigger values of the clustering algorithm.

\begin{tabular}{|c|c|c|c|c|c|c|c|c|c|c|c|}
\hline \multicolumn{3}{|c|}{ Source Beam Angulations $\left({ }^{\circ}\right)$} & \multicolumn{4}{|c|}{ Source Beam Filtrations (mm) } & \multicolumn{3}{|c|}{ Source Beam Position (cm) } & \multicolumn{2}{|c|}{ Source Beam Apertures $\left({ }^{\circ}\right)$} \\
\hline Primary Angle & Secondary Angle & Energy $(\mathrm{kVp})$ & $\mathrm{Al}$ & $\mathrm{Cu}$ & $\mathrm{Be}$ & $\mathrm{W}$ & $\mathrm{x}$ & $\mathrm{y}$ & $\mathrm{z}$ & Polar & Azimuthal \\
\hline 0.2 & 0.2 & 5 & 0.2 & 0.2 & 0.2 & 0.2 & 0.5 & 0.5 & 0.5 & 0.5 & 0.5 \\
\hline
\end{tabular}


covering a total region of $12 \times 12 \mathrm{~cm}^{2}$. The relative position between the $\mathrm{X}$-ray source and the RA phantom for the PA and PALAO irradiation setups is shown in Figs. 3 and 4.

\section{Organ dose measurement set-up}

Three clinical procedures were undertaken, namely, procedures Set1, Set 2 and Set3. They consisted of 108, 25 and 25 events, respectively. The RA phantom is made up of a set of slices. Each slice contains small perforated cavities where TLD detectors can be inserted. For this experiment, TLD detectors were placed within 5 different slices in both the right and left lung and at the level of the esophagus. TLD detectors were placed in slices 14 to 18 , which correspond approximately to the region of the heart. In Fig. 5, slices 14 to 18 are shown together with the cavities perforated within them. The cavities with a red circle correspond to those cavities where a TLD detector was placed. Furthermore, the number associated with each TLD detector is shown next to the red circle and was used to identify the TLD detectors in order to subsequently compare the measured dose with the corresponding simulated dose at the TLD detector level. During these procedures, Gafchromic films $\left(25.4 \times 30.48 \mathrm{~cm}^{2}\right.$ XR-RV3, Ashland, USA) were used to evaluate the skin dose distribution.

\section{PYMCGPU-IR simulations}

PyMCGPU-IR was used to reproduce the measurements described in Sections 2.2.1 and 2.2.2. As explained in Section 2.1, PyMCGPU-IR uses the Radiation Dose Structured Report (RDSR) to calculate doses.

\section{Patient phantom}

The current version of PyMCGPU-IR implements the Rex and Regina anthropomorphic phantoms to model a male and female patient, respectively. However, the experiments were carried out with the RA phantom. For the skin dose set-up (section 2.2.1) this is not a limitation. The skin of the Rex and Regina phantoms is segmented as a separate material and, thus, PyMCGPU-IR can calculate the peak skin dose on the skin of the REX and compare it with the measured peak skin dose on the RA phantom. Given that the RA phantom represents a male patient, we decided to use the Rex phantom. The REX phantom was not rescaled in this study and the dimensions are the ones presented in section 2.1. For

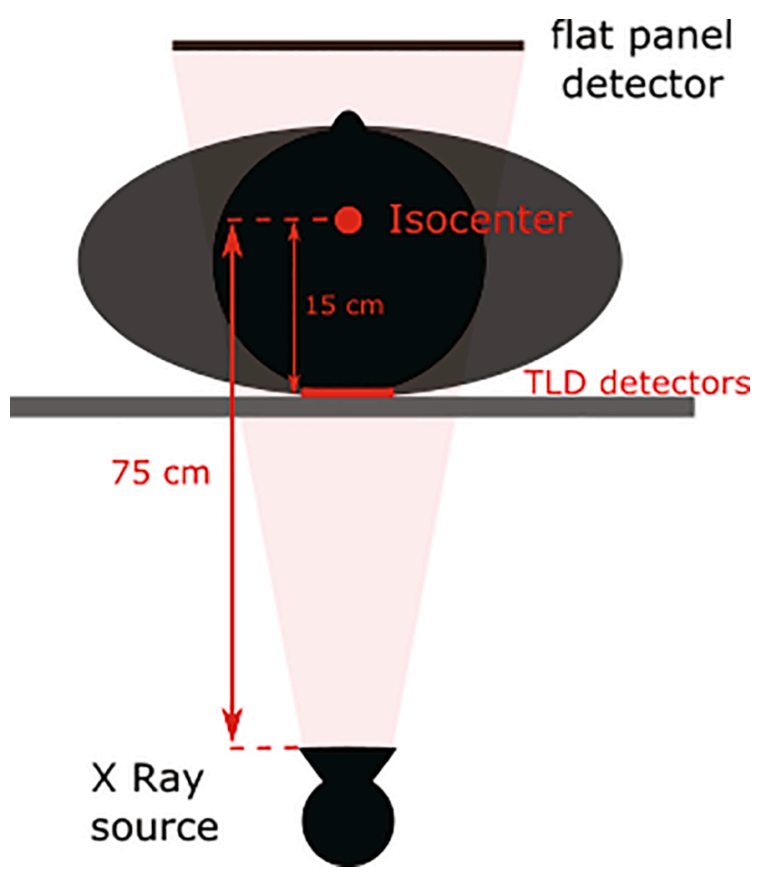

Fig. 3. PA irradiation set-up.



Fig. 4. PALAO irradiation set-up.

the organ dose calculation (section 2.2.2), in order to be able to make a comparison both as complete and as accurate as possible, the voxelized RA phantom was employed in PyMCGPU-IR.

The voxel model of the RA phantom was derived from CT images of the phantom used in the measurements [21]. The voxelized RA phantom has $256 \times 361 \times 185$ voxels of dimensions $0.15 \times 0.1 \times 0.5 \mathrm{~cm}^{3}$ and it is segmented into 5 different materials: air, soft tissue, bone and lung tissue and air-cavities. The small cavities for the TLD detectors have a volume of $0.11 \mathrm{~cm}^{3}$, and are considered to consist of air. Before implementing the voxelized RA phantom in PyMCGPU-IR we first modified it to have a new version in which the cavities shown in Fig. 5 are attributed different indexes (all made of air) in order to automatically calculate the dose in each cavity with PyMCGPU-IR.

For the skin dose set-up the clustering algorithm was not applied because the irradiation consisted of one, two and three differentiated events for the PA, PALAO and PALAOCRA set-ups, respectively. For the clinical procedures, the clustering algorithm of PyMCGPU-IR was applied (see Section 3.1 on the clustering algorithm performance).

\section{Computational resources}

In this work we used two NVIDIA GeForce 1080Ti 11 GB GPUs and an Intel ${ }^{\circledR} \_$Xeon ${ }^{\circledR} \_$E5-2670 v3 CPU, $4 \times 16$ GB RDIMM, $2133 \mathrm{MHz}$. The CUDA version used was 8.0 and the MPI version was 1.10.7. Simulations were run on an in-house computer cluster. However, the program can be executed on other machines compatible with MCGPU-IR, including laptops and clouds machines.

\section{Uncertainty evaluation}

Uncertainties of measurements are calculated according to the recommendations of the Guide to the Expression of Uncertainty in Measurement (GUM) [22]. All sources of uncertainty associated with a given measurement are evaluated and combined to obtain the range of dispersion of the measurand. In order to have a confidence level of approximately $95 \%$, an expanded uncertainty is calculated by multiplying the standard uncertainty by a coverage factor, $k$, equal to 2 . An uncertainty of $10 \%$ for $k=2$ for the experimental KAP measurement was considered and has been included in the calculation of the uncertainty of the simulation results. The uncertainty associated with the TLD 


\section{Slice 14}

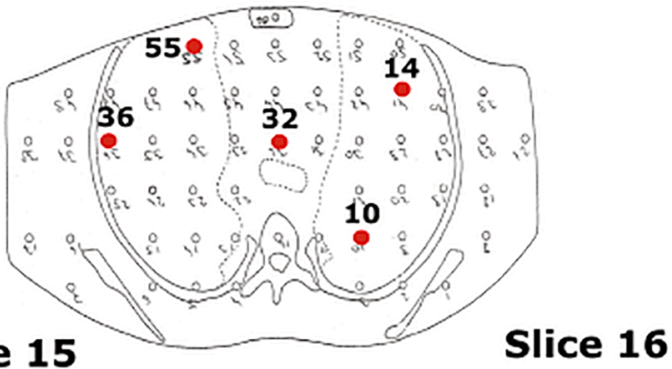

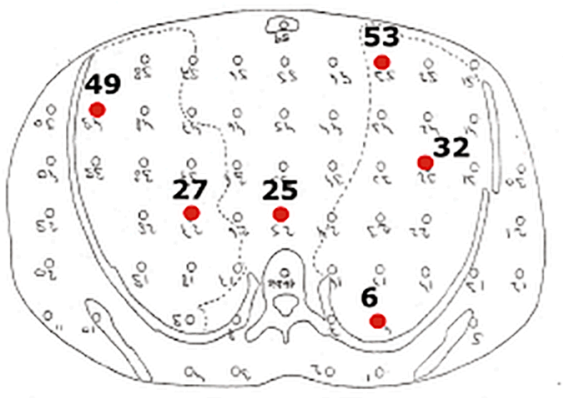

Slice 17

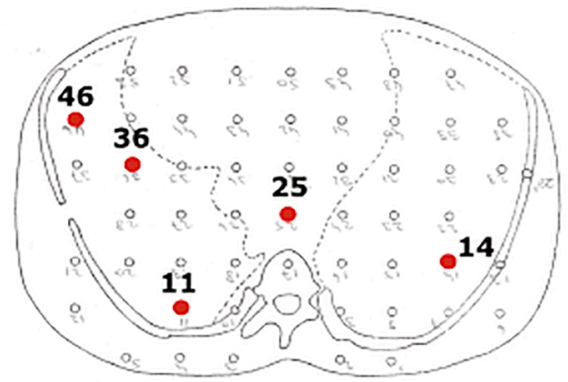

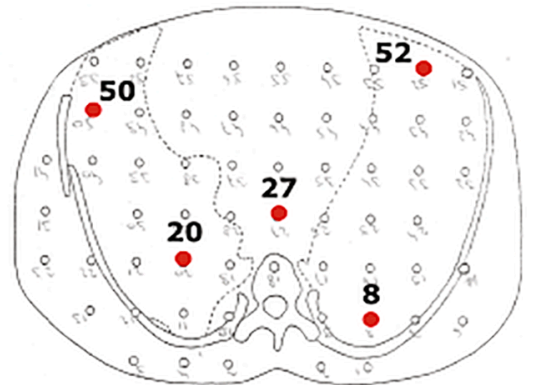

Slice 18




the associated number for identification purposes.

measurements is estimated to be $20 \%$ for $k=2$. This account for the characteristics of the TLD (mainly energy and angle dependence), their calibration and the reading system as described in [20] for exposure conditions encountered in IR.

\section{Results}

\section{Clustering algorithm performance}

In order to check the performance of PyMCGPU-IR clustering algorithm, organ doses for the three clinical procedures were calculated both with and without the clustering algorithm. Namely, the mean skin dose, the PSD, the mean heart dose and the mean lung dose were calculated. Table 2 shows the difference between the calculated doses both with and without clustering as well as the number of events simulated for each case. Differences are, for all cases, below $3 \%$, which is acceptable

Table 2

Clustering algorithm performance.

\begin{tabular}{|c|c|c|c|c|c|c|}
\hline \multirow[t]{2}{*}{ Procedure } & \multicolumn{2}{|c|}{ \# events } & \multirow{2}{*}{$\begin{array}{l}\text { Mean } \\
\text { Skin Dose } \\
\text { Diff (\%) }\end{array}$} & \multirow{2}{*}{$\begin{array}{l}\text { PSD } \\
\\
\text { Diff } \\
(\%)\end{array}$} & \multirow{2}{*}{$\begin{array}{l}\text { Mean } \\
\text { Heart } \\
\text { Dose } \\
\text { Diff (\%) }\end{array}$} & \multirow{2}{*}{$\begin{array}{l}\text { Mean } \\
\text { Lung Dose } \\
\text { Diff (\%) }\end{array}$} \\
\hline & Full & Clustered & & & & \\
\hline Set1 & 108 & 11 & $0.0 \%$ & $2.0 \%$ & $0.2 \%$ & $0.1 \%$ \\
\hline Set2 & 25 & 8 & $-0.4 \%$ & $2.7 \%$ & $-1.8 \%$ & $-1.5 \%$ \\
\hline Set3 & 25 & 7 & $0.0 \%$ & $1.7 \%$ & $2.7 \%$ & $0.8 \%$ \\
\hline
\end{tabular}

compared with the uncertainty of the experimental KAP. We also checked clustering performance with other procedures and obtained similar results.

Results for the skin dose set-up

Table 3 shows the measured and calculated peak skin dose (PSD) together with the associated uncertainties for the first set of measurements explained in Section 2.2.1. Since there were only 3 TLDs and they were all homogeneously irradiated in the PA irradiation set-up, the mean value was taken as the PSD in the results. For the PALAO and PALAOCRA irradiation set-ups, the maximum TLD dose measurement from the array of 25 TLDs was taken as the experimental PSD.

The differences between calculations and TLD measurements for the PSD varied within $\pm 6 \%$, and were consistent with the associated uncertainty. As an example, Fig. 6 shows the skin dose distribution for the PALAOCRA irradiation set-up.

\section{Results for the organ dose measurement set-up}

Results from the three clinical procedures are presented below. Set1 corresponded to 108 events that were reduced to 11, after applying the clustering algorithm. The calculation time was $15 \mathrm{~min}$. Set 2 corresponded to 25 events reduced to 8 calculations. The calculation time was $12 \mathrm{~min}$. Set3 corresponded to 25 events which were reduced to $7 \mathrm{cal}-$ culations. The calculation time was $10.5 \mathrm{~min}$.

We had both the experimental and simulated doses for each TLD. In 
Table 3

Results for the skin dose experiment.

\begin{tabular}{|c|c|c|c|c|c|c|c|}
\hline \multirow[t]{2}{*}{ Projections } & \multicolumn{2}{|c|}{ TLD measurements } & \multicolumn{2}{|c|}{ PyMCGPU-IR } & \multicolumn{2}{|l|}{ Ratio } & \multirow[t]{2}{*}{ Exec. Time (s) } \\
\hline & PSD (mGy) & $\mathrm{U}(\%)(\mathrm{k}=2)$ & PSD (mGy) & $\mathrm{U}(\%)(\mathrm{k}=2)$ & PyMCGPU-IR/TLD & $\mathrm{U}(\%)(\mathrm{k}=2)$ & \\
\hline PA & 337 & $20 \%$ & 356 & $7 \%$ & 1.06 & $21 \%$ & 90 \\
\hline PALAO & 512 & $20 \%$ & 481 & $15 \%$ & 0.94 & $25 \%$ & 180 \\
\hline PALAOCRA & 727 & $20 \%$ & 742 & $12 \%$ & 1.02 & $23 \%$ & 270 \\
\hline
\end{tabular}

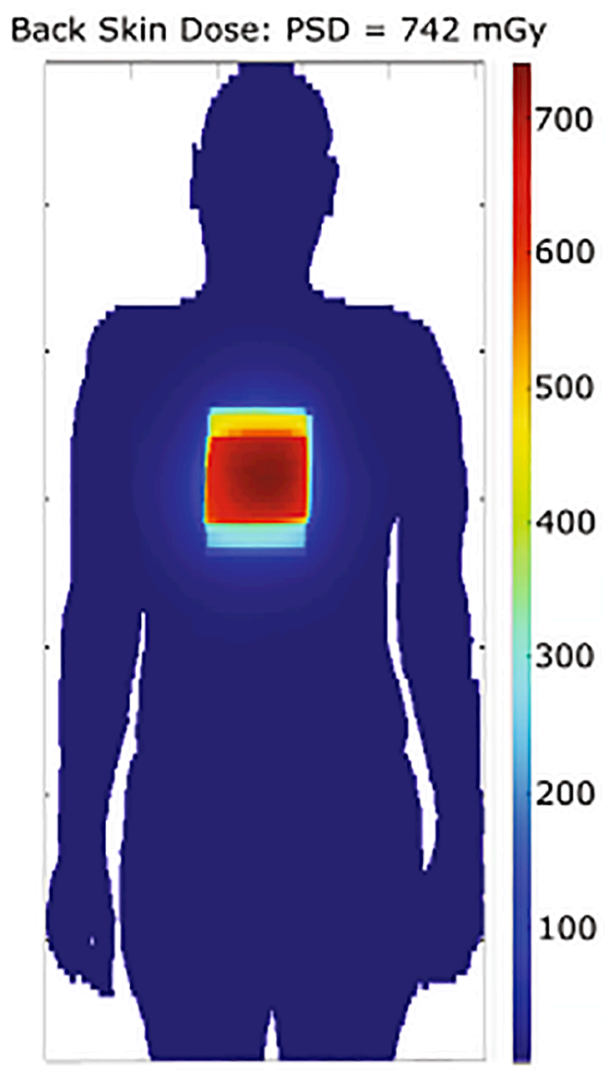

Fig. 6. Skin dose distribution for the PALAOCRA (PA + LAO $20^{\circ}+$ CRAN $15^{\circ}$ ) irradiation set-up. Voxel size of $0.21 \times 0.21 \times 0.80 \mathrm{~cm}^{3}$.

order to make the comparison as accurate and as understandable as possible, the TLDs are presented per organ (left lung, right lung and esophagus) and per slice. Both the experimental and simulated doses at the already mentioned levels were calculated as the mean dose of the corresponding TLDs. In Tables 4, 5 and 6, we present the measured and calculated absorbed dose as an average per slice separately for each organ, the associated uncertainties and the ratio between calculation and measurement. We also present the average of the organ dose calculated as the mean value of the different slices. The TLD measurement of the right lung for set 3 was lost during reading and thus the comparison of this point is not available.

Differences between calculated and measured doses are within $20 \%$ for Set1, Set2 and the left lung in Set3. However, in Set3, the dose calculation for the right lung in slice 18 and the esophagus in the 5 slices is of the order of a factor of 2 . It is believed that this difference is associated with the difficulty in correctly reproducing the position of the phantom in the calculations. Roser et al. [23] performed a study to quantify the uncertainty in organ-equivalent dose in interventional radiology due to marginal displacements of the phantom. They found that longitudinal translations of $\pm 25 \mathrm{~mm}$ induced differences ranging from $6 \%$ to $135 \%$ in the most irradiated organs, whereas the skin dose was almost constant, with a maximum difference of $5 \%$.
Table 4

Comparison of the measured mean dose of the left lung, right lung and the esophagus with the corresponding calculated doses for Set1.

\begin{tabular}{|c|c|c|c|c|c|c|c|}
\hline \multicolumn{2}{|l|}{ Set1 } & \multicolumn{2}{|c|}{$\begin{array}{l}\text { TLD } \\
\text { measurements }\end{array}$} & \multicolumn{2}{|c|}{ PyMCGPU-IR } & \multicolumn{2}{|c|}{$\begin{array}{l}\text { Calculation/ } \\
\text { Measurement }\end{array}$} \\
\hline Organ & Slice & $\begin{array}{l}\text { dose } \\
\text { (mGy) }\end{array}$ & $\begin{array}{l}\mathrm{U}(\%) \\
(\mathrm{k}= \\
2)\end{array}$ & $\begin{array}{l}\text { dose } \\
\text { (mGy) }\end{array}$ & $\begin{array}{l}\mathrm{U}(\%) \\
(\mathrm{k}= \\
2)\end{array}$ & Ratio & $\begin{array}{l}\mathrm{U}(\%) \\
(\mathrm{k}= \\
2)\end{array}$ \\
\hline \multirow[t]{6}{*}{ Left Lung } & 14 & 9 & $15 \%$ & 8 & $55 \%$ & 0.87 & $57 \%$ \\
\hline & 15 & 15 & $15 \%$ & 13 & $15 \%$ & 0.85 & $22 \%$ \\
\hline & 16 & 76 & $17 \%$ & 90 & $8 \%$ & 1.20 & $19 \%$ \\
\hline & 17 & 115 & $14 \%$ & 108 & $6 \%$ & 0.94 & $15 \%$ \\
\hline & 18 & 94 & $17 \%$ & 91 & $7 \%$ & 0.96 & $18 \%$ \\
\hline & Average & 62 & $9 \%$ & 62 & $4 \%$ & 1.00 & $9 \%$ \\
\hline \multirow{6}{*}{$\begin{array}{l}\text { Right } \\
\text { Lung }\end{array}$} & 14 & 14 & $17 \%$ & 9 & $30 \%$ & 0.69 & $34 \%$ \\
\hline & 15 & 22 & $16 \%$ & 48 & $9 \%$ & 2.13 & $18 \%$ \\
\hline & 16 & 27 & $19 \%$ & 20 & $14 \%$ & 0.76 & $23 \%$ \\
\hline & 17 & 18 & $20 \%$ & 16 & $24 \%$ & 0.92 & $31 \%$ \\
\hline & 18 & 28 & $19 \%$ & 27 & $13 \%$ & 0.98 & $23 \%$ \\
\hline & Average & 23 & $8 \%$ & 24 & $7 \%$ & 1.12 & $11 \%$ \\
\hline \multirow[t]{6}{*}{ Esophagus } & 14 & 10 & $20 \%$ & 14 & $22 \%$ & 1.41 & $30 \%$ \\
\hline & 15 & 23 & $20 \%$ & 35 & $12 \%$ & 1.53 & $23 \%$ \\
\hline & 16 & 51 & $20 \%$ & 58 & $13 \%$ & 1.14 & $24 \%$ \\
\hline & 17 & 58 & $20 \%$ & 72 & $12 \%$ & 1.25 & $23 \%$ \\
\hline & 18 & 70 & $20 \%$ & 74 & $12 \%$ & 1.06 & $23 \%$ \\
\hline & Average & 42 & $10 \%$ & 51 & $6 \%$ & 1.20 & $12 \%$ \\
\hline
\end{tabular}

Table 5

Comparison of the measured mean dose of the left lung, right lung and the esophagus with the corresponding calculated doses for Set2.

\begin{tabular}{|c|c|c|c|c|c|c|c|}
\hline \multicolumn{2}{|l|}{ Set2 } & \multicolumn{2}{|c|}{$\begin{array}{l}\text { TLD } \\
\text { measurements }\end{array}$} & \multicolumn{2}{|c|}{ PyMCGPU-IR } & \multicolumn{2}{|c|}{$\begin{array}{l}\text { Calculation/ } \\
\text { Measurement }\end{array}$} \\
\hline Organ & Slice & $\begin{array}{l}\text { dose } \\
\text { (mGy) }\end{array}$ & $\begin{array}{l}\mathrm{U}(\%) \\
(\mathrm{k}= \\
2)\end{array}$ & $\begin{array}{l}\text { dose } \\
\text { (mGy) }\end{array}$ & $\begin{array}{l}\mathrm{U}(\%) \\
(\mathrm{k}= \\
2)\end{array}$ & Ratio & $\begin{array}{l}\mathrm{U}(\%) \\
(\mathrm{k}= \\
2)\end{array}$ \\
\hline \multirow[t]{6}{*}{ Left Lung } & 14 & 18 & $15 \%$ & 19 & $25 \%$ & 1.07 & $29 \%$ \\
\hline & 15 & 10 & $14 \%$ & 11 & $14 \%$ & 1.10 & $20 \%$ \\
\hline & 16 & 95 & $15 \%$ & 95 & $7 \%$ & 1.00 & $17 \%$ \\
\hline & 17 & 137 & $14 \%$ & 124 & $5 \%$ & 0.90 & $15 \%$ \\
\hline & 18 & 118 & $16 \%$ & 95 & $7 \%$ & 0.81 & $17 \%$ \\
\hline & Average & 75 & $8 \%$ & 69 & $4 \%$ & 0.91 & $9 \%$ \\
\hline \multirow{6}{*}{$\begin{array}{l}\text { Right } \\
\text { Lung }\end{array}$} & 14 & 11 & $16 \%$ & 6 & $36 \%$ & 0.54 & $39 \%$ \\
\hline & 15 & 44 & $15 \%$ & 51 & $9 \%$ & 1.18 & $17 \%$ \\
\hline & 16 & 18 & $18 \%$ & 18 & $14 \%$ & 0.99 & $23 \%$ \\
\hline & 17 & 16 & $20 \%$ & 14 & $24 \%$ & 0.90 & $31 \%$ \\
\hline & 18 & 22 & $18 \%$ & 37 & $11 \%$ & 1.66 & $22 \%$ \\
\hline & Average & 22 & $8 \%$ & 25 & $6 \%$ & 1.14 & $10 \%$ \\
\hline \multirow[t]{6}{*}{ Esophagus } & 14 & 15 & $20 \%$ & 16 & $26 \%$ & 1.03 & $33 \%$ \\
\hline & 15 & 31 & $20 \%$ & 35 & $15 \%$ & 1.13 & $25 \%$ \\
\hline & 16 & 51 & $20 \%$ & 60 & $11 \%$ & 1.19 & $23 \%$ \\
\hline & 17 & 59 & $20 \%$ & 65 & $12 \%$ & 1.10 & $23 \%$ \\
\hline & 18 & 64 & $20 \%$ & 71 & $12 \%$ & 1.10 & $23 \%$ \\
\hline & Average & 44 & $10 \%$ & 49 & $6 \%$ & 1.12 & $11 \%$ \\
\hline
\end{tabular}

In order to analyze the influence of the positioning of the phantom, calculations for Set3 were repeated moving the position of the phantom $7.5 \mathrm{~mm}$ along the $\mathrm{x}$-axis (transversal direction) and $15 \mathrm{~mm}$ in the $\mathrm{z}$-axis (longitudinal direction). Table 7 presents the results shown in Table 6 
Table 6

Comparison of the measured mean dose of the left lung, right lung and the esophagus with the corresponding calculated doses for Set3.

\begin{tabular}{|c|c|c|c|c|c|c|c|}
\hline \multicolumn{2}{|l|}{ Set3 } & \multicolumn{2}{|c|}{ TLD measurements } & \multicolumn{2}{|l|}{ PyMCGPU-IR } & \multicolumn{2}{|c|}{ Calculation/Measurement } \\
\hline Organ & Slice & dose (mGy) & $\mathrm{U}(\%)(\mathrm{k}=2)$ & dose (mGy) & $\mathrm{U}(\%)(\mathrm{k}=2)$ & Ratio & $\mathrm{U}(\%)(\mathrm{k}=2)$ \\
\hline \multirow[t]{6}{*}{ Left Lung } & 14 & 26 & $16 \%$ & 13 & $53 \%$ & 0.49 & $55 \%$ \\
\hline & 15 & 9 & $13 \%$ & 9 & $16 \%$ & 0.98 & $21 \%$ \\
\hline & 16 & 87 & $16 \%$ & 100 & $9 \%$ & 1.16 & $19 \%$ \\
\hline & 17 & 107 & $15 \%$ & 136 & $6 \%$ & 1.28 & $16 \%$ \\
\hline & 18 & 89 & $16 \%$ & 106 & $8 \%$ & 1.19 & $18 \%$ \\
\hline & Average & 64 & $8 \%$ & 73 & $4 \%$ & 1.15 & $9 \%$ \\
\hline \multirow[t]{6}{*}{ Right Lung } & 14 & 10 & $15 \%$ & 5 & $40 \%$ & 0.48 & $43 \%$ \\
\hline & 15 & 53 & $15 \%$ & 64 & $11 \%$ & 1.21 & $18 \%$ \\
\hline & 16 & 12 & $17 \%$ & 13 & $15 \%$ & 1.13 & $23 \%$ \\
\hline & 17 & Not available & Not available & Not calculated & Not calculated & Not calculated & Not calculated \\
\hline & 18 & 17 & $18 \%$ & 30 & $12 \%$ & 1.80 & $22 \%$ \\
\hline & Average & 18 & $10 \%$ & 28.08 & $7 \%$ & 1.22 & $12 \%$ \\
\hline \multirow[t]{6}{*}{ Oesophagus } & 14 & 12 & $20 \%$ & 8 & $29 \%$ & 0.66 & $35 \%$ \\
\hline & 15 & 23 & $20 \%$ & 34 & $16 \%$ & 1.52 & $26 \%$ \\
\hline & 16 & 28 & $20 \%$ & 61 & $12 \%$ & 2.17 & $24 \%$ \\
\hline & 17 & 30 & $20 \%$ & 63 & $13 \%$ & 2.09 & $24 \%$ \\
\hline & 18 & 28 & $20 \%$ & 60 & $13 \%$ & 2.11 & $24 \%$ \\
\hline & Average & 24 & $9 \%$ & 45 & $7 \%$ & 1.86 & $11 \%$ \\
\hline
\end{tabular}

Table 7

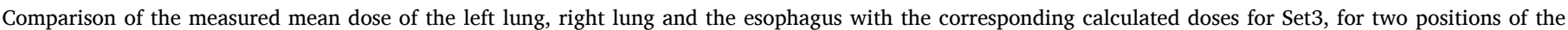
phantom.

\begin{tabular}{|c|c|c|c|c|c|c|c|}
\hline Set3 & & TLD measurement & $\mathrm{x}=0, \mathrm{z}=0$ & $\mathrm{x}=-7.5 \mathrm{~mm}, \mathrm{z}=15 \mathrm{~mm}$ & & & \\
\hline Organ & Slice & dose (mGy) & dose (mGy) & dose (mGy) & $\operatorname{diff}(\%)$ & Ratio & $\mathrm{U}(\%)(\mathrm{k}=2)$ \\
\hline \multirow[t]{6}{*}{ Left Lung } & 14 & 26 & 13.06 & 2 & $61 \%$ & 0.80 & $42 \%$ \\
\hline & 15 & 9 & 8.68 & 9 & $9 \%$ & 1.06 & $26 \%$ \\
\hline & 16 & 87 & 100.28 & 128 & $28 \%$ & 1.48 & $22 \%$ \\
\hline & 17 & 107 & 136.46 & 150 & $10 \%$ & 1.41 & $21 \%$ \\
\hline & 18 & 89 & 105.78 & 121 & $15 \%$ & 1.36 & $21 \%$ \\
\hline & Average & 64 & 72.85 & 86 & $18 \%$ & 1.35 & $11 \%$ \\
\hline \multirow[t]{6}{*}{ Right Lung } & 14 & 10 & 4.96 & 7 & $46 \%$ & 0.70 & $37 \%$ \\
\hline & 15 & 53 & 64.36 & 82 & $28 \%$ & 1.54 & $22 \%$ \\
\hline & 16 & 12 & 13.21 & 12 & $-17 \%$ & 0.94 & $26 \%$ \\
\hline & 17 & Not available & Not available & Not calculated & Not calculated & Not calculated & Not calculated \\
\hline & 18 & 17 & 29.80 & 16 & $-46 \%$ & 0.98 & $26 \%$ \\
\hline & Average & 23 & 28.08 & 29 & $4 \%$ & 1.27 & $15 \%$ \\
\hline \multirow[t]{6}{*}{ Esophagus } & 14 & 12 & 8.13 & 9 & $13 \%$ & 0.74 & $37 \%$ \\
\hline & 15 & 23 & 34.21 & 32 & $-6 \%$ & 1.42 & $25 \%$ \\
\hline & 16 & 28 & 61.07 & 39 & $-36 \%$ & 1.39 & $24 \%$ \\
\hline & 17 & 30 & 63.27 & 49 & $-23 \%$ & 1.61 & $24 \%$ \\
\hline & 18 & 28 & 60.02 & 45 & $-25 \%$ & 1.58 & $25 \%$ \\
\hline & Average & 24 & 45.34 & 35 & $-23 \%$ & 1.43 & $12 \%$ \\
\hline
\end{tabular}

for Set3 with the new simulated doses together with an additional column with the differences between the new simulated doses with those in Table 6. The ratio between simulated and experimental doses is computed with the new configuration. The simulations get slightly better with ratios below 1.6 compared with a factor of 2.17 in the first case. Differences between the new and the original simulated doses for each slice and organ differ within $60 \%$ while if we compare the averaged doses for each organ the differences are within $20 \%$. Therefore, minimal displacements can lead to high differences at the TLD level, while the doses at the organ level remain similar.

Figure 7 shows the dose distribution for clinical procedure set2 together with the Gafchromic film result for clinical procedure Set2. Visually good agreement between the Gafchromic film and the calculated skin dose distribution can be anticipated, however, no quantitative evaluation of the film has been performed.

\section{Influence of the phantom in the calculation results}

As described in Section 2.3.1 simulations for the skin dose set-up were performed with the REX phantom. Simulations were repeated with the RA phantom in order to evaluate the influence of the phantom. It was found that differences in the PSD were within $10 \%$ for the three simple irradiation set-ups.

For the organ dose set-ups, simulations were done with the RA phantom because we needed to identify the position of the TLDs in the phantom. The calculations were repeated for the three clinical procedures using the REX phantom. In these cases, the differences obtained in the simulated PSD between both phantoms were within $20 \%$.

\section{Discussion}

The comparison between PyMCGPU-IR and the experimental set-ups PA, PALAO and PALAOCRA show very good agreement in the calculation of the peak skin dose, with differences below $6 \%$. In a recent article [24], Krajinovic et al presented and validated the SkinCare application (Open Source). For validation purposes, among other tests, they used a similar set-up to measure skin dose and reported that the doses calculated by SkinCare were within $16.9 \%$ of the measurements. Likewise, in Dabin [18] up to 9 SDC tools were tested against the same skin dose setup on the same angiographic system. The absolute differences were 




Fig. 7. Skin dose distribution for clinical procedure Set2. Figure above, MC simulation (voxel size of $0.15 \times 0.1 \times 0.5 \mathrm{~cm}^{3}$ ); Figure below, Gafchromic film.

usually larger (within $\pm 40 \%$ for all of them and below $20 \%$ for 5 of them). Greffier et al. [25] compared the results obtained by using the 3D Skin Dose Map tool integrated in DoseWatch ${ }^{\circledR}$ (Ge healthcare) against 98 abdominopelvic embolisation or planned coronary angioplasty procedures. PSD was measured by using radiochromic films and computed by RDMS (PSDRDMS) using two anthropomorphic phantoms, namely, a flat (2D) phantom and adult male or female anthropomorphic phantoms (3D, ICRP phantoms). The percentage of the difference calculations and experimental values for the PSD ranged from $-35 \%$ to $61 \%$. In another study [26] the results of NEXO[DOSE] (BRACCO) in simple phantoms showed that both calculated and measured PSD values agreed with a mean difference of $7 \% \pm 5 \%$ (with a maximum difference of $13 \%$ ).

\section{Limitations of the study}

The comparison between PyMCGPU-IR and the three clinical procedures with organ dose measurements is more challenging. The comparison between simulated and experimental doses were done at the organ level for each slice. Namely, between the left and right side of the lung and for the esophagus. Measurements are time consuming and the available time for experiments in hospital limited. Thus, the number of TLDs and the number of realistic simulated sets were limited. This should be considered when interpreting the results.

For each slice the dose at the esophagus is measured by only one TLD, while the dose at the left and right of the lung is measured by one, two or three TLDs depending on the slice. In slice 17 of the right lung for Set 3 the TLD measurement was lost in the readout. The differences between calculated and measured doses are within $20 \%$ for Set 1 , Set 2 and the left lung in Set3. However, in Set3, the differences with the experimental measurements of the dose calculation for the right lung in slice 18 and the esophagus in the five slices are of the order of a factor of 2 . These differences could be explained by the uncertainty associated with the positioning of the patient relative to the source. We have shown that introducing small displacements in the patient relative to the dose can lead to large differences up to $60 \%$ for points within the phantom, while the dose at the whole organ level shows differences below 20\%. Similar behavior was reported by Roser et al [23]. Thus, we believe that the differences found between measurement and calculations in the case of Set3 are not associated with the performance of PyMCGPU-IR but with the difficulties in centering the phantom and to the fact that there was only one TLD to assess the esophagus dose in each slice.

An important parameter for an accurate dose calculation when using Monte Carlo simulation is the phantom in the geometry input file. One should use a computational phantom as close as possible to the patient anatomy, in particular when assessing organ doses. Thus, in this study, to reduce the influence of any anatomical difference between the physical phantom used in the clinical procedures and in the calculations a voxel model of the RA phantom was used for the organ dose assessment. However, since PyMCGPU-IR implements Rex and Regina phantom, PSD was calculated using the REX and the RA phantom and it was demonstrated that results are within $10 \%$ for single projections and between $9 \%$ and $17 \%$ for the clinical procedures. These differences are considered acceptable when compared with the performance of other systems [23]. Results could be improved by applying a scaling factor to the REX phantom. Work is in progress to validate and improve the scaling algorithm.

In order to accelerate calculations, a clustering algorithm is used to identify events with very similar radiological output. Several approaches were analysed before choosing the algorithm presented in section 2.1. The study of the effect of each of the 12 variables which define a radiological event and how they influence each other in the total absorbed dose is complex since these relations are not linear.

The final choice was based on the analysis of several RDSR files and the more frequent changes between events. The application of the algorithm to the 3 clinical procedures proposed in this study resulted in a computational time reduction between a factor of 10 and 3.5 with differences in peak skin dose and organ dose below $3 \%$, so we concluded that it is satisfactory for our purpose.

\section{Conclusions}

It has been proven that PyMCGPU-IR offers both high performance and accuracy for dose assessment when compared to standard Monte Carlo codes and TLD measurements. It should also be highlighted that PyMCGPU-IR provides not only the dose values at specific positions, as in the case of the TLDs, but also the dose distribution, the position of maximum dose and the organ doses. In addition, PyMCGPU-IR overcomes the time limitations of CPU-based MC codes.

When compared to other similar software performances [18], PyMCGPU-IR presents some clear advantages as regards its validation against measurements and its independence from the vendor (provided that the required input parameters are available).

At present, the most critical point is the difficulty of knowing accurately the patient's position on the table well enough, since this highly influences the accuracy of the dose calculation. This limitation is found 
in any similar software tool. One possible approach to overcome this problem is to use one of the X-ray images to improve matching between the simulated and the experimental geometry.

At the current time, PyMCGPU-IR only provides the doses after the procedure has finished, as it uses the RDSR, which is provided after the procedure. Howerver, PyMCGPU-IR could be easily adapted to provide real-time dose calculation if the radiation source information would be provided in real-time.

\section{Declaration of Competing Interest}

The authors declare that they have no known competing financial interests or personal relationships that could have appeared to influence the work reported in this paper.

\section{Acknowledgment}

This project has received funding from the Euratom Research and Training Programme 2014-2018 under grant agreement No 755523.

\section{References}

[1] Balter S, Hopewell JW, Miller DL, Wagner LK, Zelefsky MJ. Fluoroscopically guided interventional procedures: a review of radiation effects on patients' skin and hair. Radiology 2010;254:326-41. https://doi.org/10.1148/radiol.2542082312.

[2] European Commission 2013. Council Directive (Euratom 2013/59) laying down basic safety standards for protection against the dangers arising from exposure to ionising radiation. Off. J. Eur. Commun. 2014.

[3] ICRP. Radiological Protection in Medicine. ICRP Publication 105. Ann. ICRP 2007; 37(6).

[4] Malchair F, Dabin J, Deleu M, Merce MS, Bjelac OC, Gallagher A, et al. Review of skin dose calculation software in interventional cardiology. Phys Med 2020;80: 75-87. https://doi.org/10.1016/j.ejmp.2020.09.023.

[5] Jones AK, Pasciak AS. Calculating the peak skin dose resulting from fluoroscopically-guided interventions. Part II: case studies. J Appl Clin Med Phys 2012;13:174-86. https://doi.org/10.1120/jacmp.v13i1.3693.

[6] MEDIRAD. Implications of Medical Low Dose Radiation Exposure. http://www. medirad-project.eu/ [accessed 1 July 2021].

[7] Badal A, Badano A. Accelerating Monte Carlo simulations of photon transport in a voxelized geometry using a massively parallel graphics processing unit. Med Phys 2009;36:4878-80. https://doi.org/10.1118/1.3231824.

[8] Fernández Bosman D, García Balcaza V, Delgado C, Principi S, Duch MA, Ginjaume M. Validation of the MC-GPU Monte Carlo code against the PENELOPE/ penEasy code system and benchmarking against experimental conditions for typical radiation qualities and setups in interventional radiology and cardiology. Phys Med 2021;82:64-71. https://doi.org/10.1016/j.ejmp.2021.01.075.

[9] D2.23. Accelerated Monte Carlo code for an online patient monitoring system in interventional cardiology procedures. D. Fernández-Bosman, M. Ginjaume, M.A. Duch, J. Dabin, F. Vanhavere, June 2021. In: http://www.medirad-project.eu/re sults. [accessed 1 July 2021].
[10] Python software Foundation downloadable in https://brochure.getpython.info/ [accessed 11 November 2021]

[11] NEMA PS3/ISO 12052, Digital Imaging and Communications in Medicine (DICOM) Standard, National Electrical Manufacturers Association, Rosslyn, VA, USA (available free at http://www. dicomstandard.org/).

[12] Salvat F, Fernandez-Varea JM, and Sempau J. PENELOPE 2006: A code system for monte carlo simulation of electron and photon transport. Workshop Proceedings Barcelona, Spain, 4-7 July 2006, OECD 2006 NEA No.6222, 2006.

[13] García Balcaza V, Camp A, Badal A, Andersson M, Almen A, Ginjaume M, et al. Fast Monte Carlo codes for occupational dosimetry in interventional radiology. Phys Med 2021;85:166-74. https://doi.org/10.1016/j.ejmp.2021.05.012.

[14] Siemens Healtheeners. https://www.siemens-healthineers.com/medical-imaging low-dose/right-dose-portfolio. [accessed 06 October 2021].

[15] Zankl M, et al. Voxel-based models representing the male and female ICRP reference adult - the skeleton. Radiat Prot Dosim 2007;127(1-4):174-86.

[16] Zankl M. Adult Male and Female Reference Computational Phantoms (ICRP Publication 110). Japanese J Heal Phys 2010;45.

[17] ICRP. Adult Reference Computational Phantoms. ICRP Publication 110. Ann. ICRP 2009;39(2).

[18] Dabin J, Blidéanu V, Ciraj Bjelac O, Deleu M, De Monte F, Feghali JA, et al. Accuracy of skin dose mapping in interventional cardiology: Comparison of 10 software products following a common protocol. Phys Med 2021;82:279-94. https://doi.org/10.1016/j.ejmp. 2021.02.016.

[19] Parisi A, Dabin J, Schoonjans W, Van Hoey O, Mégret P, Vanhavere F. Photon energy response of LiF:Mg, Ti (MTS) and LiF:Mg, Cu, P (MCP) thermoluminescent detectors: experimental measurements and microdosimetric modeling. Radiat Phys Chem 2019. https://doi.org/10.1016/j.radphyschem.2019.05.021.:357-69. doi: 10.5453/jhps.45.357.

[20] Kržanović N, Blideanu V, Ciraj-Bjelac O, Plagnard J, Schoonjans W, Živanović M, et al. Performance testing of dosimeters used in interventional radiology: results from the VERIDIC project. Radiat Meas 2021;141. https://doi.org/10.1016/j. radmeas.2021.106515.

[21] Struelens L, Vanhavere F, Smans K. Experimental validation of Monte Carlo calculations with a voxelized Rando-Alderson phantom: a study on influence parameters. Phys Med Biol 2008;53:5831-44. https://doi.org/10.1088/00319155/53/20/018.

[22] Joint Committee for Guides in Metrology. Evaluation of measurement data Guide to the expression of uncertainty in measurement. JCGM 100:2008 GUM 1995 with minor corrections. Available in: https://www.bipm.org/utils/common/ documents/jcgm/JCGM_100_2008_E.pdf.

[23] Roser P, Birkhold A, Zhong X, Ochs P, Stepina E, Kowarschik M, et al. Pitfalls in interventional X-ray organ dose assessment-combined experimental and computational phantom study: application to prostatic artery embolization. Int $\mathrm{J}$ Comput Assist Radiol Surg 2019;14:1859-69. https://doi.org/10.1007/s11548019-02037-6.

[24] Krajinović M, Kržanović N, Ciraj-Bjelac O. Vendor-independent skin dose mapping application for interventional radiology and cardiology. J Appl Clin Med Phys 2021;22:145-57. https://doi.org/10.1002/acm2.13167.

[25] Greffier J, Grussenmeyer-Mary N, Hamard A, Goupil J, Miller DE, Cayla G, et al. Clinical evaluation of a dose management system-integrated 3D skin dose map by comparison with radiochromic films. Eur Radiol 2020;30:5071-81. https://doi. org/10.1007/s00330-020-06877-8.

[26] Colombo PE, Rottoli F, Felisi M, De Mattia C, Riga S, Sutto M, et al. Validation of a dose tracking software for skin dose map calculation in interventional radiology. Phys Med 2020;72:122-32. https://doi.org/10.1016/j.ejmp.2020.03.008. 